\title{
Review of: "Examination of the roles of a conserved motif in the PriA helicase in structure-specific DNA unwinding and processivity"
}

\author{
Wei Yang ${ }^{1}$ \\ 1 National Institutes of Health
}

Potential competing interests: The author(s) declared that no potential competing interests exist.

Duckworth et al. report the functional importance of a flexible but conserved linker between two N-terminal DNA-binding domains (WH and 3థBD) in PriA. PriA is a DNA helicase and is essential for reloading the replicative helicase DnaB onto a collapsed DNA replication fork, thus enabling replication restart. Based on the PriA structure reported by the same group (the Keck laboratory), the conserved linker interacts with the C-terminal helicase domains, but it is $25 \AA$ from the ATPase active site. In the current paper the authors mutated three conserved residues in the linker to Ala, individually or together, and compared their DNA binding, unwinding and ATPase activities with WT PriA. As a world leader on this topic, the authors established the rationale, and designed and executed experiments well. Their results shed light on the regulatory mechanism of the PriA helicase activity. Data interpretation, however, will benefit from quantitative analysis, and conclusions may be drawn with broader consideration and alternative possibilities.

1. The linker between the $\mathrm{WH}$ and $3 \not B D$ domains by docking onto the helicase domain certainly restrain the movement of the two linked domains and thereby may regulate the relative distance and orientation of $\mathrm{WH}$ and $3 \not \mathrm{BD}$. Since the interface is predominantly hydrophobic, the linker has some flexibility to move. If DNA fork binding is reduced by the W186A mutation, the reduction of the DNAdependent ATPase and helicase activity of the mutant PriA may be explained.

2. Mutation of the most conserved residue, W186A, has obvious defects in SsDNA-dependent ATPase activity and unwinding a two-strand DNA fork. The following two points need clarification. (a) Although the authors report that DNA binding is unaltered, Fig. 2B suggests that WT and W186A PriA bind to the four-strand fork with different affinities. The analysis would be more convincing if each lane of EMSA gel is quantified. (b) In Fig. 3A and 3B, W186A mutant PriA is shown defective in unwinding the two-strand fork, but in Fig. 3C W186A and WT PriA are indistinguishable in unwinding a three-strand fork, which contains the two-strand fork. Could the authors explain why?

3. The rescue of the triple mutation by lengthening the ssDNA region in the DNA substrate, thus allowing loading more than one helicase molecule, may not be unique to PriA, but a general phenomenon for monomeric helicases. Have the authors quantified the WT and triple mutant PriA in DNA unwinding and 
compared their activities shown in Fig. 3 and 4 quantitatively? It seems that WT PriA exhibits increased helicase activity with longer SSDNA too. Is the increasing of helicase activity similar or different for WT vs. triple mutant PriA on the longer ssDNA substrate?

4. The factor(s) that restore the helicase activity of triple mutations do(es) not have to be the reason that leads to the defects of mutations. The suggestion of multiple PriA molecules loading via the linker region lacks supporting experimental evidence.

5. There are alternative explanations for the conservation of the linker and its hydrophobic groove in the PriA helicase domain. One is that the linker may be replaced by another flexible part of PriA intermolecularly as shown in Fig. 5 , in which the triple mutant seems to be more prone to form aggregates on DNA than WT PriA. The second possibility is it is replaced by a partner protein in the process of macromolecular assembly and DnaB reloading. 